2015-05

PLANT ABIOTIC STRESS

TOLERANCE ANALYSIS IN

CAULIFLOWER USING A CURD

MICROPROPAGATION SYSTEM

\title{
Rihan, Hail
}

http://hdl.handle.net/10026.1/4266

10.17660/actahortic.2015.1083.3

Acta Horticulturae

International Society for Horticultural Science (ISHS)

All content in PEARL is protected by copyright law. Author manuscripts are made available in accordance with publisher policies. Please cite only the published version using the details provided on the item record or document. In the absence of an open licence (e.g. Creative Commons), permissions for further reuse of content should be sought from the publisher or author. 


\title{
Plant Abiotic Stress Tolerance Analysis in Cauliflower Using a Curd Micropropagation System
}

\author{
H.Z. Rihan ${ }^{1}$, M. Al-Issawi ${ }^{2}$, M. Al-Shamari ${ }^{1}$, M. Elmahrouk ${ }^{3}$ and M.P. Fuller ${ }^{1}$ \\ ${ }^{1}$ School of Biomedical and Biological Sciences, Faculty of Science and Technology, \\ Plymouth University, PL4 8AA, United Kingdom \\ 2 Agriculture College, Al-Anbar University, Anbar, Iraq \\ ${ }^{3}$ Faculty of Agriculture, Kafer Elsheikh University, Egypt
}

Keywords: abiotic stress, artificial seeds, cold tolerance, meristematic clusters, plant growth regulator

\begin{abstract}
An effective protocol for cauliflower micropropagation was optimised and developed which enabled the production of tens of thousands of cauliflower microshoots from one cauliflower curd. The large number of microshoots that can be produced per culture unit facilitates the use of this protocol to analyse both the physiological and molecular components of abiotic stress tolerance. The protocol was used for cauliflower cold tolerance analysis and it was demonstrated that low temperature acclimation increased the cold tolerance of explants. The effect of two additives used with the cauliflower culture media on cold tolerance were evaluated. ABA significantly decreased both cold tolerance in acclimated and non-acclimated cauliflower microshoots whilst molybdenum had a highly positive effect on cold tolerance of cauliflower microshoots. Moreover, molybdenum had the capacity to increase the cold tolerance of cauliflower microshoots without low temperature treatment. This is the first study that confirmed this feature of molybdenum and it is believed that this finding could have an application in the field.
\end{abstract}

\section{INTRODUCTION}

An effective protocol for cauliflower micropropagation was designed by Kieffer et al. (2001) and optimised by Rihan et al. (2011, 2012a,b,c). This protocol enables the production of thousands of microshoots per cauliflower curd and provides an optimal system to analyse physiological responses in cauliflower in an in vitro micropropagation system including abiotic stress tolerance.

Plant growth and productivity are limited by both biotic and abiotic factors (Seki et al., 2003) and it has been reported that abiotic stresses can cause a decrease in yield of crops up to $50 \%$ resulting in very high economic losses (Vij and Tyagi, 2007). The world population is increasing rapidly (Mahajan and Tuteja, 2005) and therefore, the minimization of these losses is one of the main objectives for plant and crop specialists and since it is very difficult to control abiotic stress resulting from climate change and human activities, the development of stress tolerant crop genotypes is necessary (Mahajan and Tuteja, 2005).

Low temperature is one of the main abiotic stresses that affects plant growth and production globally. It limits the geographical distribution of agronomic species and significantly decreases the yield of several crops around the world (Pearce and Fuller, 2001). It is important to study frost damage mechanisms and to breed for cold tolerant cultivars since the many crops must survive exposure to occasional sub-zero temperatures and (Deane, 1994).

Cold acclimation is defined as the exposure of plants to low non-freezing temperatures leading to significant positive effect on the cold tolerance of plants (Thomashow, 1999; Smallwood and Bowles, 2002). Extensive research has been conducted to improve the understanding of the biochemical and molecular basis of the cold acclimation response and the changes that take place throughout this process but there is relatively little information about the molecular responses of cauliflower to cold.

The plant hormone ABA (abscisic acid) plays an essential role as a chemical 
signal in response to biotic and abiotic stresses such as salt, cold, drought and wounding. ABA stimulates several changes in plant physiological, molecular and developmental progressions resulting in plant adaptation to the stress environment (Ton et al., 2009). Abiotic stresses such as drought induce the synthesis of ABA which in turn leads to stomatal closure and the activation of the expression of stress related genes (Lee and Luan, 2012). It appears that ABA has an essential role in the induction of various stress signals controlling downstream stress responses and plants adjust their ABA content constantly in response to changes in physiological and environmental conditions.

Molybdenum (Mo) is an essential trace element present in the soil required by plants. Many oxidation states of molybdenum ranging from zero to VI have been reported in agricultural soils and state VI is considered to be the most excited and active (Sun et al., 2009). In planta molybdenum plays an essential role in plant reduction and oxidation reactions. Li et al. (2001) proposed that molybdenum is involved in the amelioration of wheat frost tolerance and several mechanisms, by which molybdenum can improve frost tolerance, have been suggested. However, the mechanism for this enhancement has not been definitively determined.

The aim of this study was to investigate the effect of cold acclimation on the cold tolerance of cauliflower microshoots and the effect of ABA and Mo on cold tolerance were evaluated with the microshoot culture system.

\section{MATERIAL AND METHODS}

Young plants of several cultivars of cauliflower were obtained from field trial plots courtesy of Simmonds \& Sons Ltd. (West Cornwall, Devon, UK) and replanted in raised beds at the University of Plymouth. The plants were grown according to standard commercial practice (Whitwell et al., 1982) and raised to maturity when the curds were harvested and stored at $2-4^{\circ} \mathrm{C}$ until required. The use of several cultivars gave a continuous supply of cauliflower heads over the experimental period.

Large pieces $(1-5 \mathrm{~cm})$ of curd were surface sterilized by immersion in $10 \%(\mathrm{v}: \mathrm{v})$ domestic bleach $(0.06 \%$ sodium hypochlorite), unthickened basic domestic bleach (Sainsburys, UK) for 15 min followed by a double wash with sterile distilled water. Explants were produced in a laminar flow hood by mechanically eliminating the mass of non-responsive tissue (stem branches) and shaving off the upper meristematic layer using a sterilized scalpel. The meristimatic clusters were then homogenized using a commercial blender (Waring model 800 , UK) at approximately $1700 \mathrm{rev} \mathrm{m^{-1 }}$ in maintenance S23 liquid medium (blending medium) for $30 \mathrm{~s}$. The maintenance S23 liquid medium consists of MS (Murashige and Skoog, 1962) (4.4 g L-1 Sigma, M5519-50L) + sucrose $30 \mathrm{~g} \mathrm{~L}^{-1}$. The blending medium containing the micro-explants was then sieved through precision sieves $(212,300$ and $600 \mu \mathrm{m}$ aperture) (Endecotts Ltd., London, UK). The micro-explants were collected from the sieves, weighed and converted to aliquots of explants for subsequent culture using small precision volumetric measures $(74 \mu \mathrm{l})$. 125-ml plastic pots, each containing $30 \mathrm{ml}$ of culture medium (composition dependent on the treatment) were cultured with $74 \mu \mathrm{l}$ of the $300-600 \mu \mathrm{m}$ micro-explants. The pots were constantly shaken $\left(150 \mathrm{rev} \mathrm{min}^{-1}\right)$ during culture at $20^{\circ} \mathrm{C}$ and exposed to $16 \mathrm{~h}$ photoperiod $(40 \mu \mathrm{mol}$ $\mathrm{m}^{-2} \mathrm{~s}^{-1}$ PAR light intensity). Micro-explants subsequently developed to microshoots in culture.

Cauliflower microshoot cultures ('Dionis') (26 days old) were used for the production of artificial seeds following procedures described in Rihan et al. (2011) and divided into two groups and the first acclimated at $4^{\circ} \mathrm{C}$ for 15 days and the second used as a control (grown at $20^{\circ} \mathrm{C}$ ). Frost resistance analysis of the artificial seeds was carried out to assess the effectiveness of the acclimation process. Artificial seeds were placed in sterile petri dishes with a small piece of sterile ice to ensure ice nucleation and then exposed to different temperatures sequentially $20,0,-2,-4,-6,-8$, and $-10^{\circ} \mathrm{C}$ with a $2-\mathrm{h}$ hold at each temperature in a Sanyo programmable chamber. Samples were removed at the end of the 2-h hold of each temperature. Samples were kept at $4{ }^{\circ} \mathrm{C}$ overnight to thaw and then cultivated on semi-solid maintenance media S23. The conversion rates and the 
average weights of plantlets produced were assessed after 27 days of culture. Ten replicate culture pots with four artificial seeds per pot were used with each temperature treatment.

\section{The Effect of Abscisic Acid}

Cauliflower microshoots ('Cool') were produced using the same system described above with five concentrations of ABA (Sigma, A-7631) $\left(0,0.5,1,2,4 \mathrm{mg} \mathrm{L}^{-1}\right)$ added to the culture medium to determine the effect of ABA on microshoot cold tolerance. This experiment was divided into parts: in the first, 21-day-old microshoots were tested at, 20 (control), $-4,-8$ and $-12^{\circ} \mathrm{C}$ and the frost damage assessed using a relative electrical conductivity (REC) as follows: $10 \mathrm{~g}$ of microshoots derived from each treatment were placed in boiling tubes. All the tubes were labelled in test tube racks and placed in the Sanyo freezing chamber. A small piece of ice was added to each tube to ensure ice nucleation and the chamber was programmed to fall to various low temperatures as above with a hold of two hours at each temperature after which samples were then left at $4^{\circ} \mathrm{C}$ overnight to defrost and then transferred to the laboratory bench at ambient temperature and $20 \mathrm{ml}$ distilled water was added to each tube using an automatic dispenser (Oxford, catalogue number $470 \mathrm{~A}$ ). The tubes were covered with aluminium foil and left overnight at room temperature to allow leaching of electrolytes from cells damaged by the freezing. The post-freezing electrical conductivity (EC) of each solution was measured using a conductivity meter the following day. All the samples (tubes) were then autoclaved in order to rupture all the cells to produce the total cell leachate. After autoclaving, they were kept overnight and then the post-autoclaving EC was measured for all samples. REC was calculated as follows: $\mathrm{REC} \%=$ Post-freezing EC/Post-autoclaving $\mathrm{EC} \times 100$. 3 replicates (tubes of which contained $10 \mathrm{~g}$ microshoots) were used for each ABA treatment at each temperature.

The second part of the experiment was transferred to a cold room at $4^{\circ} \mathrm{C}$ after 21 days of culture and left for 15 days for cold acclimation. Microshoot cold tolerance (frost damage) was assessed following the same procedure as above and the frost damage was analysed using the relative electrical conductivity (REC \%).

\section{The Effect of Molybdenum}

Cauliflower microshoots ('Fremont') were produced using the system described above with the exception that two concentrations of Mo (0 and $15 \mathrm{ppm})$ were used with the culture media. These concentrations were used because earlier experiments had shown that higher concentrations had negative effects on the growth and development of microshoots. Samples of 15-day-old microshoots were encapsulated in sodium alginate matrix in order to produce artificial seeds.

Frost resistant analysis of the artificial seeds was carried out to test the effect of molybdenum (Mo). The frost resistance was assessed as described above and artificial seeds were cultured on maintenance medium to assess survival. The conversion rate and the average weights of plantlets were the evaluated after 28 days of culture. Five replicate culture pots, each containing $30 \mathrm{ml}$ of maintenance S23 semi-solid media and cultured with 5 artificial seeds, were used with each treatment.

Another set of cauliflower microshoots ('Fremont') were produced with two concentrations of Mo (0 and $15 \mathrm{ppm})$ and cultured in the culture media. After 25 days growth, the shaker containing the microshoot cultures was transferred from room temperature to the cold room at $4^{\circ} \mathrm{C}$ for 15 days for acclimation. The cold tolerance of acclimated microshoots was tested as above and the REC procedure used to assess damage.

Results are presented as means + standard error (S.E.). All data were subjected to analysis of variance (ANOVA) using Minitab software (version 15) and comparisons of means were made with least significant difference test (LSD) at the $5 \%$ level of probability. 


\section{RESULTS}

\section{The Effect of Acclimation on Artificial Seed Cold Tolerance}

The conversion rate of non-acclimated artificial seeds significantly decreased after exposure to freezing temperatures lower than $-4^{\circ} \mathrm{C}$. Acclimation significantly $(\mathrm{P}<0.001)$ improved the cold tolerance of artificial seeds with conversion rates of $100 \%$ down to $-8^{\circ} \mathrm{C}$ (Fig. 1).

Acclimation had a residual effect on subsequent growth of transplanted artificial seeds and plants grew slower than the controls. Sub-zero exposure accentuated this slightly, however, below $-4^{\circ} \mathrm{C}$ growth of acclimated artificial seeds was significantly better than the controls (Fig. 2).

\section{The Effect of Abscisic Acid on the Microshoots Cold Tolerance}

Low temperature treatment significantly increased REC\% (Fig. 3) $(\mathrm{P}<0.001)$. There was no significant effect of ABA on the REC $\%$ at control $\left(20^{\circ} \mathrm{C}\right),-4$ and $-12^{\circ} \mathrm{C}$ $(\mathrm{P}=0.062, \mathrm{P}=0.133$ and $\mathrm{P}=0.870$ respectively), but a highly significant effect was observed at $-8^{\circ} \mathrm{C}(\mathrm{P}=0.003)$ where the use of $\mathrm{ABA}\left(0.5 \mathrm{mg} \mathrm{L}^{-1}\right)$ significantly increased the $\mathrm{REC} \%$ (frost damage). However, the higher the concentration of ABA the lower the increase in REC\% compared with the control. Moreover, there was no significant increase in $\mathrm{REC} \%$ between the control and $4 \mathrm{mg} \mathrm{L}^{-1}$ ABA treatments (Fig. 4). A test temperature of $-8^{\circ} \mathrm{C}$ was the most effective in demonstrating the clear effect of $\mathrm{ABA}$. Lower test temperatures than $-8^{\circ} \mathrm{C}$ significantly increased the $\mathrm{REC} \%$ in all $\mathrm{ABA}$ treatments.

\section{The Effect of Abscisic Acid on the Cold Tolerance of Acclimated Microshoots}

There was no significant effect of $\mathrm{ABA}$ on acclimated microshoot cold tolerance (REC\%) when these microshoots were treated at $20^{\circ} \mathrm{C}$ (Control), -4 and $-12^{\circ} \mathrm{C}(\mathrm{P}=0.214$, $\mathrm{P}=0.062$ and $\mathrm{P}=0.870$ ). However, a significant effect of $\mathrm{ABA}$ on acclimated microshoots frost damage was observed when microshoots were treated at $-8^{\circ} \mathrm{C}$. ABA significantly increased REC\% (frost damage) at this temperature $(\mathrm{P}=0.003)$. As before, $-8^{\circ} \mathrm{C}$ was confirmed to be the sensitive temperature where the effect of ABA was observed (Fig. 5).

\section{The Effect of Molybdenum on the Cold Tolerance of Artificial Seeds of Cauliflower}

Molybdenum had significantly positive effect on artificial seed cold tolerance at both -10 and $-12^{\circ} \mathrm{C}$ treatments $(\mathrm{P}<0.001)$. Whilst the conversion rates decreased significantly at temperatures lower than $-8^{\circ} \mathrm{C}$ for the controls those treated with Mo tolerated low temperature down to $-12^{\circ} \mathrm{C}$ (Fig. 6).

\section{The Effect of Molybdenum Treatment on the Cold Tolerance of Acclimated Cauliflower Microshoots}

The use of $15 \mathrm{ppm}$ of Mo significantly improved the cold tolerance of acclimated microshoots $(\mathrm{P}=0.022)$ at $-10^{\circ} \mathrm{C}($ Fig. 7$)$.

\section{DISCUSSION}

Cold acclimation which is defined as the exposure of plants to low, non-freezing temperature, has been reported to increase the cold tolerance in several plant species (Shinozaki and Yamaguchi-Shinozaki, 1996; Thomashow, 1999, 2001; Gilmour et al., 2000; Jan et al., 2009). In terms of Brassica olearaceae var. botrytis, the experiment reported here demonstrated the capacity of cauliflower microshoots to be cold acclimated and that acclimation was also able to increase cauliflower artificial seed cold tolerance and this confirms findings with in vivo grown cauliflower (Fuller, 1993; Deane, 1994). Cold acclimation causes several biochemical and physiological changes in plant tissues including an increase in the sugars, proline and soluble protein concentrations. It also embraces some modifications in the lipid composition of the cell membranes. These changes culminate in the effect of cold acclimation significantly improve the cold tolerance of plants (Thomashow, 1999; McKhann et al., 2008). The use of a liquid culture 
micropropagation system as described here for cauliflower facilitates the application of various chemical materials with this media in order to assess the characteristics of microshoots produced and consequently the quality of cauliflower artificial seeds. It was thought that $\mathrm{ABA}$ could be an important material for improving the microshoots abiotic stress (cold) since ABA is an essential controller of plant growth and development and is an important regulator for stress resistance (Kempa et al., 2008; Fait et al., 2008). Hirayama and Shinozaki (2007) proposed that ABA accumulates in response to various abiotic stresses, for example: cold, drought and high salinity. ABA is commonly considered an essential element involved in intermediating different environmental stresses and plants react to different conditions with overlapping but separate physiological mechanisms. Therefore, it is logical to suppose that an ABA-dependent signalling pathway acts in concert with ABA-independent stress specific pathways to express suitable metabolic response (Kempa et al., 2008). The use of ABA with cauliflower culture media however was found to have negative effects on microshoots cold tolerance when the microshoots were treated at $-8^{\circ} \mathrm{C}$. The frost damage was higher in the ABA treated microshoots compared with non-treated microshoots. Knight (2004) reported that water relations during the growing period may be the effective element that influences the response of plants to ABA and its capacity to up-regulate CBF genes. They also mentioned that whether or not the plants have been cultured on soil or tissue culture might critically affect their sensitivity to ABA. However, the mechanism by which ABA apparently decreases the cold tolerance of cauliflower microshoots needs further investigation.

The application of Mo improved the cold tolerance of cauliflower artificial seeds (microshoots) without acclimation at $4^{\circ} \mathrm{C}$ and enhanced the cold tolerance of acclimated cauliflower microshoots. Other reports have indicated that molybdenum could have a role in the amelioration of frost damage (Du et al., 1994; Li et al., 2001) and freezing (AlIssawi et al., 2013; Sun et al., 2006a) and several hypothesises have been examined to investigate the Mo effect mechanism on plant cold tolerance. Sun et al. (2006a) mentioned that Mo could increase the anti-oxidative defence in plants by increasing the activity of the anti-oxidative enzymes such as SOD, CAT and POX resulting in an increase in plant cold tolerance. This is argued because cold stress normally induces oxidation stresses, producing Reactive Oxygen Species (ROS) such as superoxide radicals $\left(. \mathrm{O}_{2}\right)$, hydroxyl radicals $(. \mathrm{OH})$ and hydrogen peroxidase $\left(\mathrm{H}_{2} \mathrm{O}_{2}\right)$. ROS makes complexes with DNA, lipids and proteins causing cellular damage (Sattler et al., 2000).

\section{CONCLUSION}

This study reported the efficiency of the cauliflower microshoot micropropagation system for assessing cauliflower cold stress tolerance and confirmed the functionality of the cold response mechanism in microshoots. The use of Mo in the culture medium was confirmed to have a significant effect on the cold acclimation of acclimated and nonacclimated cauliflower microshoots. The use of ABA in the culture medium was confirmed to have a neutral or negative effect on cold acclimation of microshoots.

\section{Literature Cited}

Al-Issawi, M., Rihan, H.Z., Woldie, W.A., Burchett, S. and Fuller, M.P. 2013. Exogenous application of molybdenum affects the expression of CBF14 and the development of frost tolerance in wheat. Plant Physiol Biochem. 63:77-81.

Deane, C. 1994. Whole plant and tissue culture studies on frost tolerance in cauliflower. Ph.D., The National University of Ireland.

Du, Y.Q., Wang, Y.H., Wei, W.X. and Wang, Z.R. 1994. Effect of molybdenum fertilizer application on the nitrogen and molybdenum nutritions of wheat. Journal of Huazhong Agriculture 13(4):384-389.

Fait, A., Fromm, H., Walter, D., Galili, G. and Fernie, A.R. 2008. Highway or byway: the metabolic role of the GABA shunt in plants. Trends Plant Sci. 13:14-19.

Fuller, M.P. 1993. Varietal differences in frost hardiness of cauliflower. Aspects of 
Applied Biology 34:179-182.

Gilmour, S.J., Sebolt, A.M., Salazar, M.P., Everard, J.D. and Thomashow, M.F. 2000. Overexpression of the Arabidopsis CBF3 transcriptional activator mimics multiple biochemical changes associated with cold acclimation. Plant Physiol. 124:1854-1865.

Hirayama, T. and Shinozaki, K. 2007. Perception and transduction of abscisic acid signals: keys to the function of the versatile plant hormone ABA. Trends Plant Sci. 12:343351.

Jan, N., Mahboob Ul, H. and Andrabi, K.I. 2009. Cold resistance in plants: a mystery unresolved. Electron J. Biotechn. 12(3):1-15.

Kempa, S., Krasensky, J., Dal Santo, S., Kopka, J. and Jonak, C. 2008. A central role of abscisic acid in stress-regulated carbohydrate metabolism. Plos One 3:3935.

Kieffer, M., Simkins, N., Fuller, M.P. and Jellings, A.J. 2001. A cost effective protocol for in-vitro mass propagation of cauliflower. Plant Science 160:1015-1024.

Knight, H., Zarka, D.G., Okamoto, H., Thomashow, M.E. and Knight, M.R. 2004. Abscisic acid induces CBF gene transcription and subsequent induction of coldregulated genes via the CRT promoter element. Plant Physiol. 135:1710-1717.

Lee, S.C. and Luan, S. 2012. ABA signal transduction at the crossroad of biotic and abiotic stress responses. Plant, Cell \& Environment 35:53-60.

Li, W., Wang, Z., Mi, G., Han, X. and Zhang, F. 2001. Molybdenum deficiency in winter wheat seedlings as enhanced by freezing temperature. J. Plant Nutr. 24:1195-1203.

Mahajan, S. and Tuteja, N. 2005. Cold, salinity and drought stresses: an overview. Arch. Biochem. Biophys. 444:139-158.

Mckhann, H., Gery, C., Berard, A., Leveque, S., Zuther, E., Hincha, D., De Mita, S., Brunel, D. and Teoule, E. 2008. Natural variation in $C B F$ gene sequence, gene expression and freezing tolerance in the Versailles core collection of Arabidopsis thaliana. BMC Plant Biol. 8:105.

Pearce, R.S. and Fuller, M.P. 2001. Freezing of barley studied by infrared video thermography. Plant Physiol. 125:227-240.

Rihan, H.Z., Al Shamari, M. and Fuller, M.P. 2012a. The production of cauliflower microshoots using curd meristematic tissues and hypocotyl-derived callus. Acta Hort. 961:427-434.

Rihan, H.Z. Al-Issawi, M., Al-Swedi, F. and Fuller, M.P. 2012b. The effect of using PPM (plant preservative mixture) on the development of cauliflower microshoots and the quality of artificial seed produced. Sci. Hort. 141:47-52.

Rihan, H.Z., Al-Issawi, M., Burchett, S. and Fuller, M.P. 2011. Encapsulation of cauliflower (Brassica oleracea var. botrytis) microshoots as artificial seeds and their conversion and growth in commercial substrates. PCTOC 107:243-250.

Rihan, H.Z., Al-Issawi, M., Burchett, S. and Fuller, M.P. 2012c. Artificial seed production from encapsulated microshoots of cauliflower (Brassica oleraceae var. botrytis). Acta Hort. 961:419-425.

Sattler, U., Calsou, P., Boiteux, S. and Salles, B. 2000. Detection of oxidative base DNA damage by a new biochemical assay. Arch. Biochem. Biophys. 376:26-33.

Seki, M., Kamei, A., Yamaguchi-Shinozaki, K. and Shinozaki, K. 2003. Molecular responses to drought, salinity and frost: common and different paths for plant protection. Curr. Opin. Biotechnol. 14:194-199.

Shinozaki, K. and Yamaguchi-Shinozaki, K. 1996. Molecular responses to drought and cold stress. Curr. Opin. Biotechnol. 7:161-167.

Smallwood, M. and Bowles, D.J. 2002. Plants in a cold climate. Philosophical Transactions of the Royal Society B: Biological Sciences 357(1423):831-847.

Sun, X., Hu, C. and Tan, Q. 2006a. Effects of molybdenum on antioxidative defense system and membrane lipid peroxidation in winter wheat under low temperature stress. Journal of Plant Physio. and Molecular Biol. 32:175.

Sun, X., Hu, C., Tan, Q., Liu, J. and Liu, H. 2009. Effects of molybdenum on expression of cold-responsive genes in abscisic acid (ABA)-dependent and ABA-independent pathways in winter wheat under low-temperature stress. Ann Bot. 104(2):345-356. 
Thomashow, M.F. 1999. Plant cold acclimation: freezing tolerance genes and regulatory mechanisms. Annu. Rev. Plant Physiol. Plant Mol. Biol. 50:571-599.

Thomashow, M.F. 2001. So what's new in the field of plant cold acclimation? Lots! Plant Physiol. 125:89-93.

Ton, J., Flors, V. and Mauch-Mani, B. 2009. The multifaceted role of ABA in disease resistance. Trends Plant Sci. 14:310-317.

Vij, S. and Tyagi, A.K. 2007. Emerging trends in the functional genomics of the abiotic stress response in crop plants. Plant Biotechnol J. 5:361-380.

Yu, M., Hu, C. and Wang, Y. 1999. Influences of seed molybdenum and molybdenum application on nitrate reductase activity, shoot dry matter, and grain yields of winter wheat cultivars. J. Plant Nutr. 22:1433-1441.

\section{Figures}

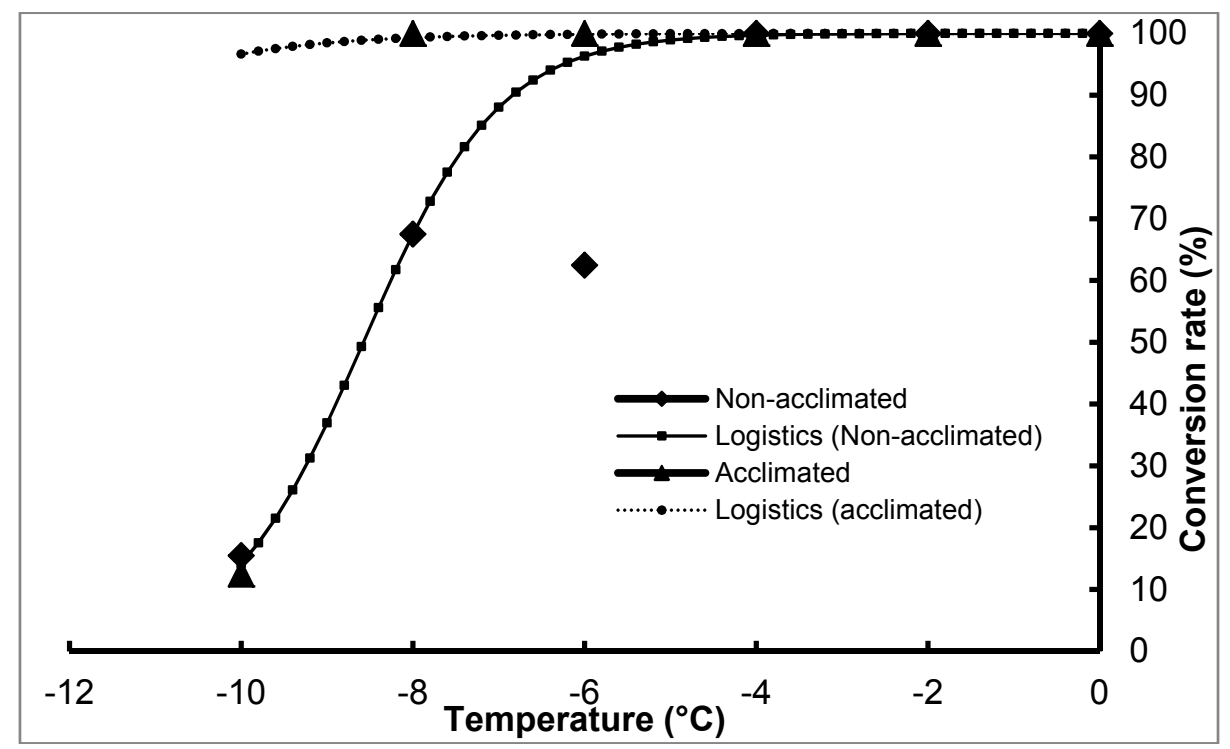

Fig. 1. The effect of cold acclimation on artificial seeds cold tolerance assessed by their conversion rate at different low temperatures $(\mathrm{LSD}=10.11)$. 


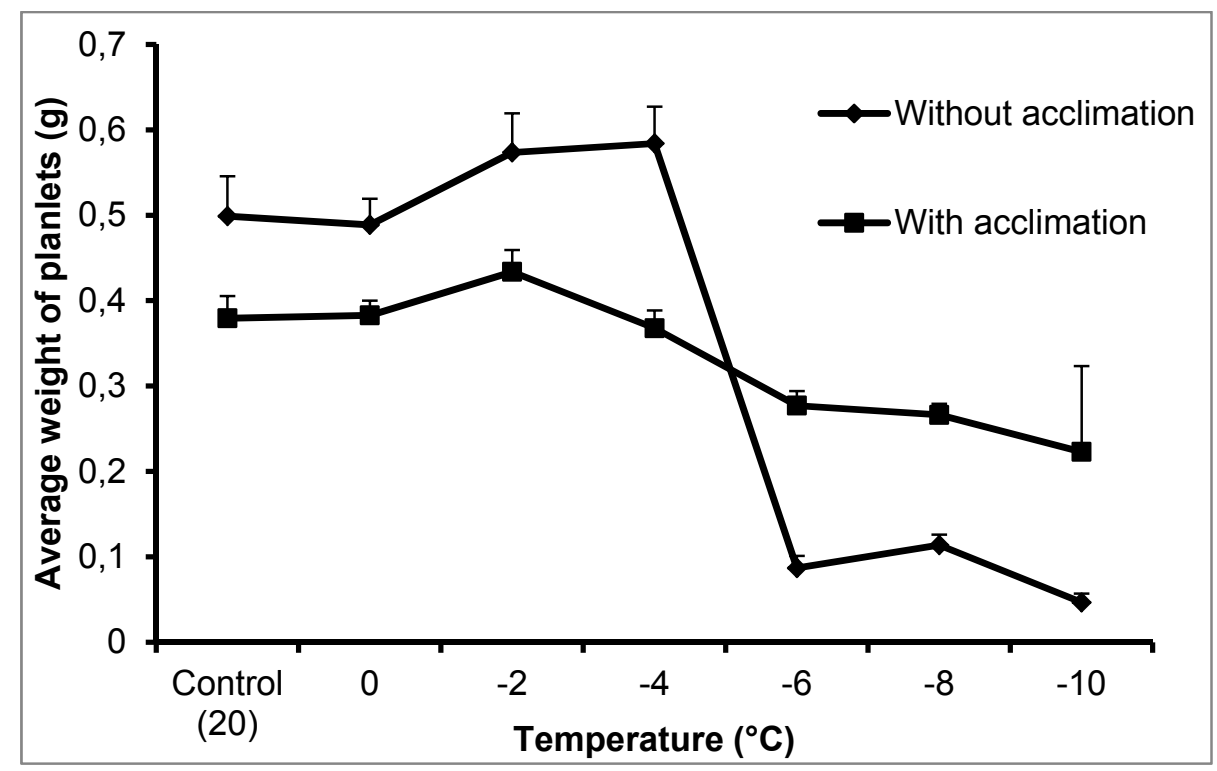

Fig. 2. The effects of temperature treatments on the average weights of cauliflower plantlets produced from both acclimated and non-acclimated artificial seeds $(\mathrm{LSD}=0.0885)$.

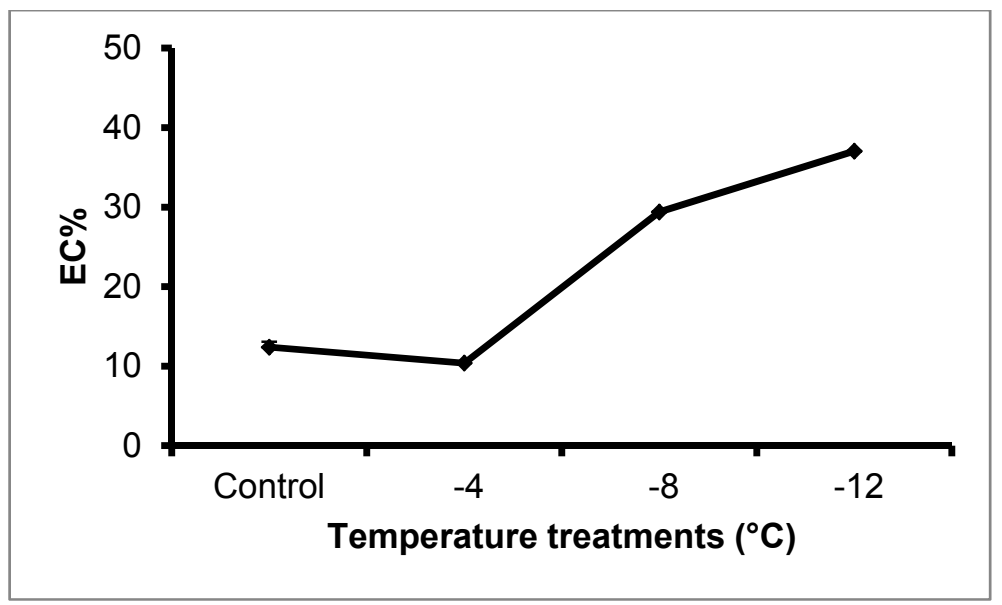

Fig. 3. The effect of temperature treatments on the relative REC\% (frost damage) in cauliflower microshoots (LSD=4.53). 


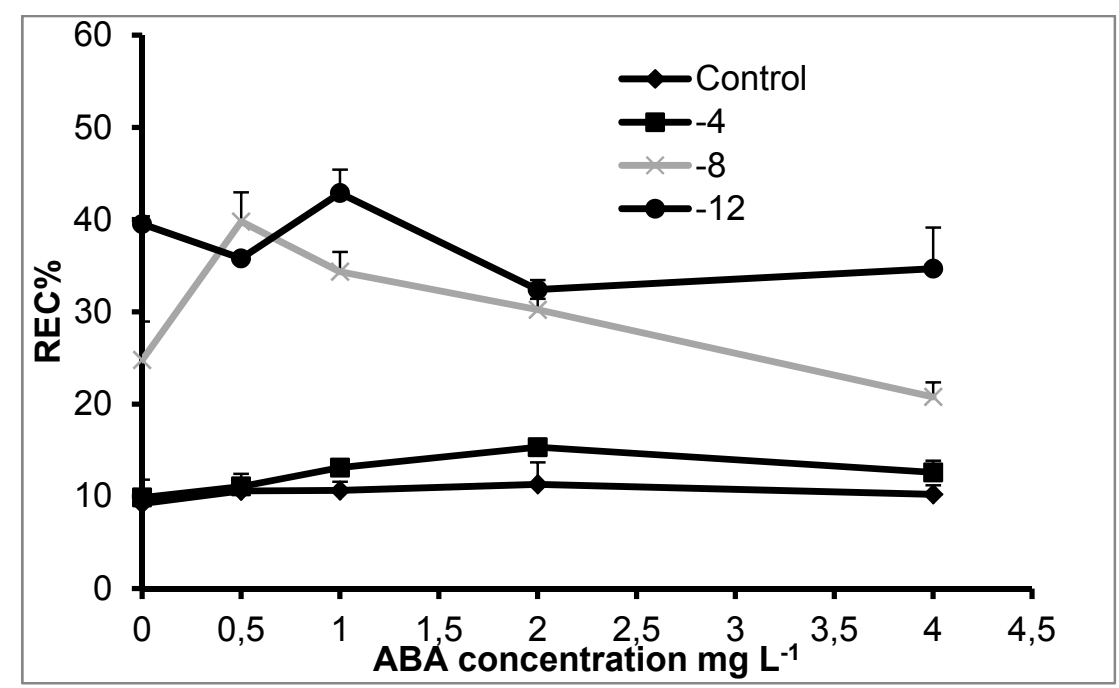

Fig. 4. The effect of ABA on the REC \% of microshoots treated with 20 (Control), $-4,-8$, and $-12^{\circ} \mathrm{C}\left(\mathrm{LSD}\right.$ for $\mathrm{REC} \%$ at $\left.-8^{\circ} \mathrm{C}=6.7\right)$.

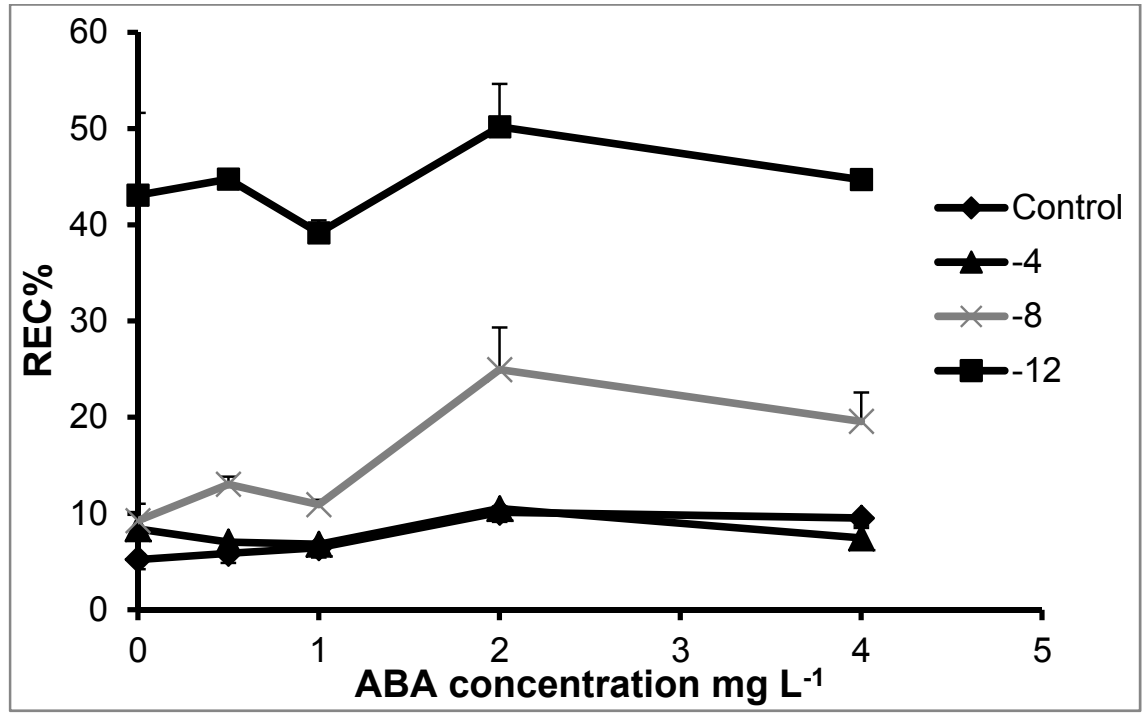

Fig. 5. The effect of ABA on the REC \% of microshoots treated with $20,-4,-8$, and $-12{ }^{\circ} \mathrm{C}$ (LSD for the $-8^{\circ} \mathrm{C}$ treatment $=0.056$ ). 


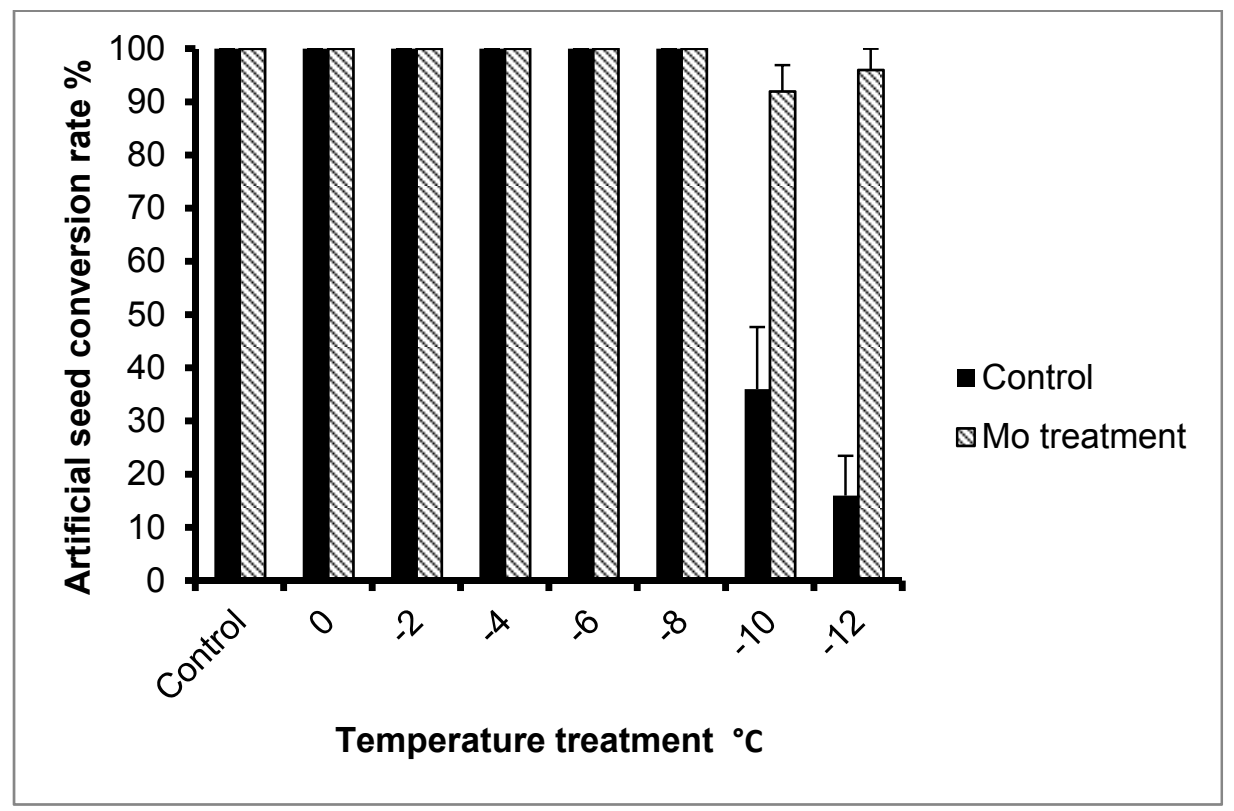

Fig. 6. The effect of using 15 PPM of Mo with microshoots culture media on the artificial seeds cold tolerance $(\mathrm{LSD}=25.78)$.



Fig. 7. The effect of Mo treatment on the cold tolerance assessed as EC\% of acclimated cauliflower microshoots $\left(\mathrm{LSD}=4.30\right.$ at $\left.-10^{\circ} \mathrm{C}\right)$. 